\title{
Valor pronóstico del PET/CT en cáncer pulmonar. Estudio de sobrevida y caracterización metabólica tumoral
}

\author{
DAVID LADRÓN DE GUEVARA, ANÍBAL FUENTES ${ }^{\mathrm{a}}$, \\ CYRO FARIÑA ${ }^{\mathrm{a}}$, CAMILO CORRAL ${ }^{\mathrm{a}}$, RAÚL PEFAUR
}

\section{Prognostic value of PET/CT in lung cancer. Retrospective analysis of 47 patients}

\begin{abstract}
Background: PET/CT (Positron emission tomography/computed tomography) is a hybrid image modality widely used in oncology, for staging, therapy evaluation or follow up. Aim: To evaluate the prognostic value of PET/CT in lung cancer. Material and Methods: Retrospective review of PET/CT records, selecting 51 patients with a lung malignancy, mass or nodule referred for PET/CT between December 2008 and December 2010. All had pathological confirmation of malignancy and had not been treated previously. Age, gender, body mass index, radiological features of lung tumor and metastases, and lung tumor ${ }^{18} \mathrm{~F}$-fluoro-2-deoxy-d-glucose uptake using the SUV (Standardized uptake value) index were recorded. Survival was analyzed using Kaplan-Meier curves and a Cox proportional regression analysis. Results: Pathology confirmed the presence of lung cancer in 47 patients aged 30 to 88 years. Four patients (7.8\%) had other type of tumors such as carcinoid or lymphoma. Fifty percent of lung cancer patients died during a mean observation lapse of 18 months (range: 2-34 months). Patients with metastases, local lymph node involvement, a lung tumor size $\geq 3 \mathrm{~cm}$ and high tumor uptake (SUVmax > 6) had significantly lower survival. Occurrence of metastases was the only independent prognostic factor in the Cox regression. A lung lesion with a SUVmax $\geq 12$ was always associated to hilar/mediastinal lymph node involvement. Conclusions: PET/CT imaging gives important prognostic information in lung cancer patients.
\end{abstract}

(Rev Med Chile 2013; 141: 41-48).

Key words: Lung neoplasms; Medical oncology; Positron-emission tomography and computed tomography; Prognosis.

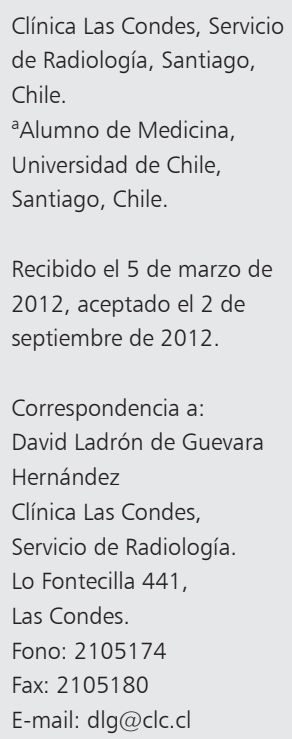

$\mathrm{E}$ 1 PET/CT (Positron emission tomography/ computed tomography) con F18-FDG (F18fluorodeoxiglucosa) es una técnica de imagen híbrida, que combina un examen de medicina nuclear de carácter funcional como es el PET con otro estudio radiológico eminentemente morfológico como es la tomografía computada (TC). Su uso en oncología ha aumentado geométricamente en los últimos años, particularmente en el estudio de cáncer pulmonar y nódulo pulmonar solitario.

El radiofármaco más empleado en oncología es el F18-Fluorodeoxiglucosa (F18-FDG), que corresponde a una molécula de glucosa marcada con Flúor-18, un isótopo emisor de positrones. El alto consumo de azúcar que muestra gran parte de las neoplasias malignas permite detectar con alta sensibilidad lesiones primarias y secundarias, y la relación entre el grado de captación y la agresividad y velocidad de crecimiento tumoral proporciona información acerca de la biología tumoral como un verdadero "marcador biológico"

El valor pronóstico del PET/CT en cáncer 
pulmonar ha sido explorado en varias publicaciones, tanto en el estudio inicial y etapificación como en la respuesta metabólica al tratamiento, demostrándose una relación inversa entre la captación tumoral de glucosa marcada y la sobrevida de los pacientes ${ }^{2}$.

El objetivo de este estudio es evaluar el valor pronóstico del PET/CT con F18-FDG en pacientes con cáncer pulmonar, y compararlo con otros factores pronósticos clínicos e imagenológicos.

\section{Pacientes y Método}

\section{Pacientes}

Se revisaron retrospectivamente todos los estudios PET/CT de cuerpo entero efectuados en el Servicio de Radiología de Clínica Las Condes desde diciembre de 2008 hasta diciembre de 2010, seleccionando aquellos que fueron derivados por cáncer pulmonar en etapificación, o con diagnóstico de tumor, masa, nódulo pulmonar en estudio que tuvieron una biopsia posterior concordante con malignidad. Se excluyó todo paciente con tratamiento previo (cirugía, quimioterapia, radioterapia) o con otra malignidad conocida para evitar incluir metástasis pulmonares de otro primario en el estudio.

\section{Adquisición y cuantificación del PET/CT}

Todos los PET/CT fueron efectuados en un equipo GE Discovery STE de 16 canales, con adquisición de CT para corrección de atenuación y localización, seguido por adquisición $3 \mathrm{D}$ de imágenes metabólicas para el mismo campo. Los PET/CT de cuerpo entero se realizaron $60 \mathrm{~min}$ después de la inyección de F18-FDG calculada según de peso $(\mathrm{kg})$.

Se llevó a cabo una entrevista con cada paciente para conocer sus antecedentes y dar las indicaciones de preparación para el examen. Dicha entrevista fue realizada por un médico o un tecnólogo médico, según la disponibilidad.

Antes de administrar la dosis ev de F18-FDG se midió glicemia capilar mediante hemoglucotest en todos los pacientes, con valor de corte de 150 $\mathrm{mg} / \mathrm{dl}$. En caso de niveles mayores se instaló suero fisiológico para hidratación, y se controló glicemia hasta alcanzar un nivel adecuado.

Se cuantificó la captación del tumor primario pulmonar en todos los casos, utilizando el índice SUVmax (Standarized uptake value maximum), el que se obtiene dibujando un área de interés sobre la lesión, con cálculo automático de la actividad del vóxel más captante, resultando en una medida de actividad por volumen corregida por peso del paciente según la siguiente fórmula (A):

SUV:

Actividad en una lesión (Bq) / Volumen vóxel (ml) Actividad inyectada (Bq) / Peso pcte (g)

\section{Factores pronósticos estudiados}

El peso, talla, índice de masa corporal, hábito tabáquico, antecedentes de cáncer previo fueron obtenidos de la entrevista y evaluación previa al examen. Se revisaron las imágenes de los PET/ CT de pacientes con cáncer pulmonar, midiendo el diámetro máximo del tumor primario, su captación utilizando índice SUVmax, y evaluando el compromiso ganglionar hiliar y mediastínico y presencia de metástasis a distancia. Se calculó un índice de captación relativa mediante la fórmula SUVmax/diámetro, que traduciría el grado de captación como un valor independiente del tamaño. El resultado histopatológico se clasificó según tipo tumoral y grado de diferenciación celular.

\section{Tratamiento}

Los pacientes con cáncer pulmonar células no pequeñas fueron manejados según criterios quirúrgicos guiado por clasificación TNM, con intento de cirugía curativa hasta etapa III en los casos que no tenían contraindicación médica. Todos los pacientes etapa IV fueron manejados con terapia sistémica. En el caso de cáncer pulmonar células pequeñas sólo uno con enfermedad limitada fue sometido a lobectomía, y el resto se manejó con esquemas de quimio y radioterapia.

\section{Sobrevida}

Sólo se incluyó a pacientes con cáncer pulmonar histológicamente confirmado. Los pacientes con otros tumores (carcinoide, linfoma) y sin histología completa fueron excluídos. El estado vital y causa de muerte de todos los pacientes fueron consultados en el Registro Civil, con un tiempo de observación promedio de 18 meses (rango 2-34 meses). Se consideró fallecido aquel paciente con causa de muerte asociada directa o indirectamente al cáncer pulmonar. Los fallecimientos por causa no ligada al cáncer fueron observados como vivos hasta la fecha de defunción. 


\section{Análisis estadístico}

Se construyeron curvas de sobreviva utilizando prueba de Kaplan-Meier y regresión proporcional de Cox para todos los factores mencionados. Para el análisis estadístico se utilizó test de Student, comparación de proporciones y Fisher`s exact test (programa estadístico MedCalc). Para el estudio de correlación se utilizó test Spearman Rank Order. Un valor de $\mathrm{p}<0,05$ fue considerado significativo.

\section{Resultados}

El 92,2\% (47/51) de los pacientes correspondió a cáncer pulmonar (edad promedio 64,7 años, rango 30-88 años), y $7,8 \%(4 / 51)$ a otros tumores (carcinoide, linfoma). Del primer grupo, en 3 casos el estudio anatomopatológico no determinó el tipo histológico o diferenciación del cáncer pulmonar, por lo que fueron excluidos del estudio pronóstico.

Los promedios y rangos de tamaño y captación tumoral, y la frecuencia de compromiso ganglionar y metastático según diagnóstico se muestran en la Tabla 1.

No se encontró diferencias significativas entre edad, sexo, tamaño tumoral, frecuencia de compromiso ganglionar local ni existencia de metástasis a distancia entre los tipos de neoplasias. Al comparar el grupo cáncer pulmonar células no pequeñas (CPCNP) con el grupo cáncer pulmonar células pequeñas (CPCP), tampoco se encontraron diferencias en estas variables.

Se observó una correlación entre el diámetro máximo tumoral y la captación tumoral expresada como SUVmax ( $r: 0,762, p<0,0001$ ). La Figura 1 grafica dicha correlación según el tipo histológico del cáncer pulmonar y según el estado vital.

El adenocarcinoma presentó una captación de F18-FDG significativamente más baja $(\mathrm{p}<0,05)$ que el carcinoma escamoso (CE) (menor SUVmax y

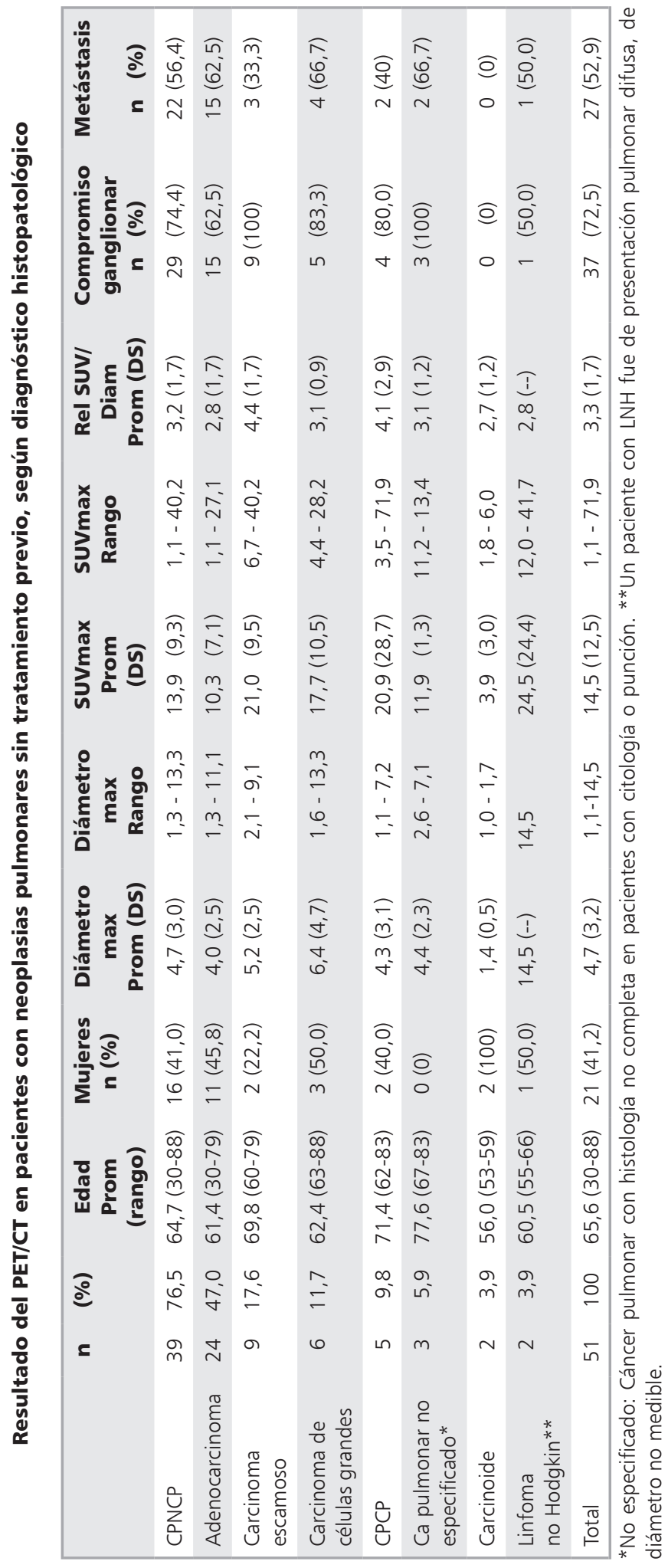




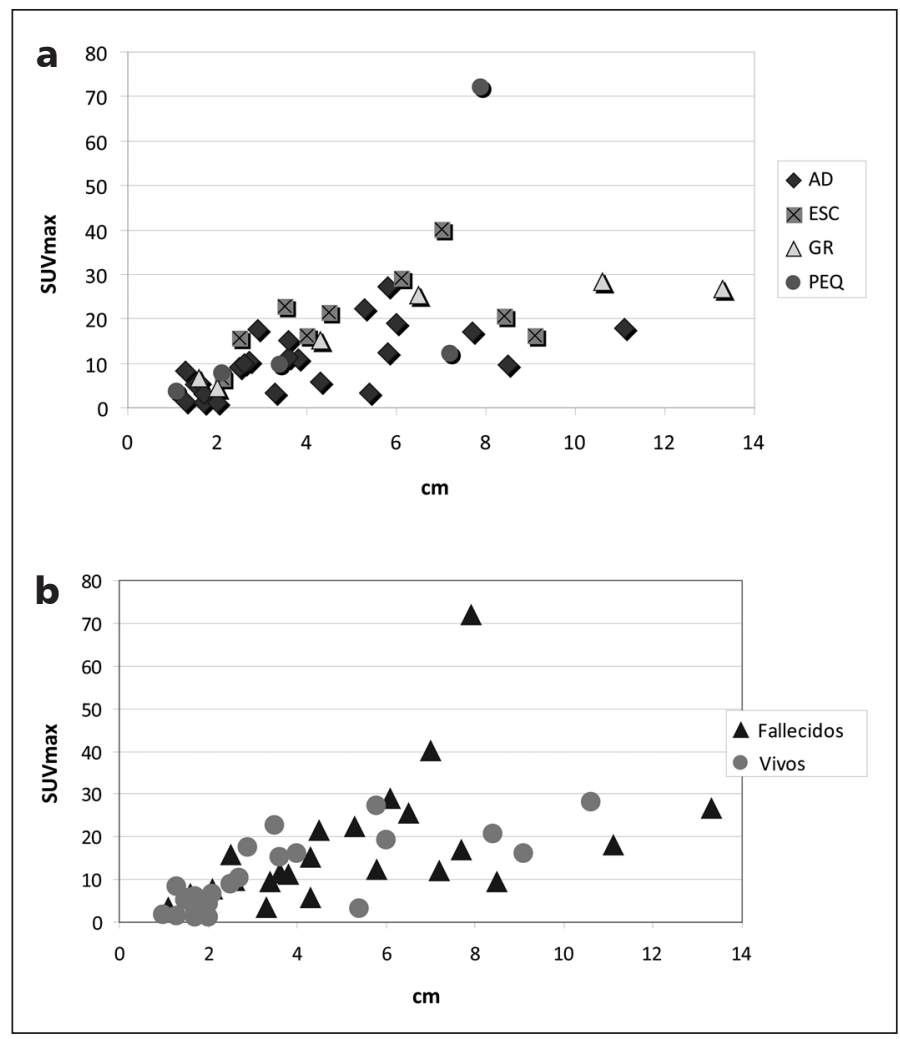

Figura 1. Correlación entre captación (SUVmax) y tamaño tumoral según el tipo histológico (a) del cáncer pulmonar y según el estado vital (b).

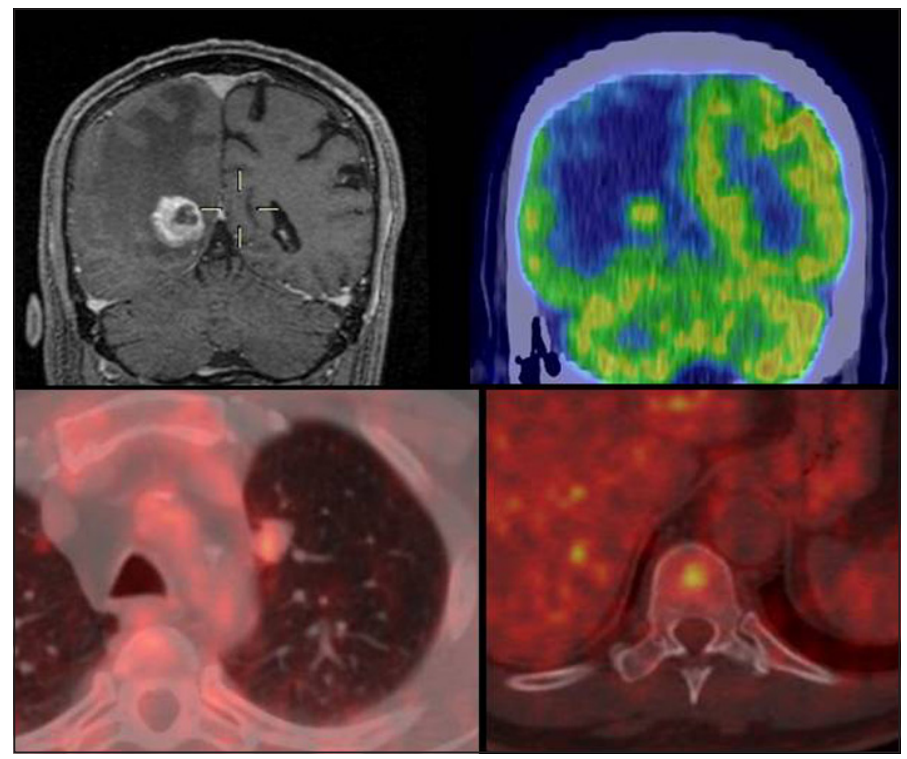

Figura 2. Paciente de 71 años, gran fumador detenido, con un adenocarcinoma pulmonar moderadamente diferenciado de 1,7 cm en LSI (foto inferior izquierda). Debuta con metástasis cerebral (RM y PET/CT en imágenes superiores) y óseas (sólo se muestra en columna vertebral). menor relación SUVmax/diámetro) y que el carcinoma de células grandes (CCG) (menor SUVmax), sin diferencias significativas en sus tamaños.

De los pacientes con cáncer pulmonar que evidenciaron metástasis a distancia, 91,7\% (22/24) presentaban compromiso ganglionar hiliar o mediastínico concomitante. Sin embargo, dos pacientes sin adenopatías locales mostraron siembra secundaria. Uno correspondió a un adenocarcinoma $(1,7 \mathrm{~cm}$, SUVmax: $3,1)$ que debutó con metástasis cerebrales -que luego fueron irradiadas- y que al PET/CT mostró además metástasis óseas (Figura 2). El segundo paciente tenía un adenocarcinoma bronquioloalveolar (según la clasificación actual adenocarcinoma invasivo) de 5,4 $\mathrm{cm}$ y SUVmax de 3,2 , con metástasis y derrame pleural hipercaptante. Ninguno falleció en el período de observación.

Dos pacientes con cáncer pulmonar (2/47) tuvieron otro cáncer concomitante detectado por el PET/ $\mathrm{CT}$, uno correspondió a una lesión polipoidea maligna de colon (adenocarcinoma tubular) y otro a un cáncer escamoso de cabeza y cuello (seno piriforme).

Los pacientes sin diseminación ganglionar ni metástasis tuvieron tumores de significativo menor tamaño y captación [diámetro promedio: 1,8 $\mathrm{cm}$ (DS: $0,7 \mathrm{~cm}$ ), SUVmax promedio: 4,7 (DS: 3,5 )] que aquellos con enfermedad diseminada [diámetro promedio: 5,3 cm (DS: 2,9), SUVmax promedio: 17,3 (DS: 1,8)]. No hubo diferencias significativas de tamaño y captación tumoral entre los pacientes con compromiso ganglionar vs aquellos con metástasis a distancia.

Todos los pacientes con tumores pulmonares con $S U V \max \geq 12$, y $94 \%$ (31/33) de aquellos con SUVmax $\geq$ 6 , tuvieron enfermedad ganglionar hiliar y/o mediastínica (Tabla 2). En cambio, el SUVmax no fue capaz de 


\section{Compromiso ganglionar regional y ocurrencia de metástasis según captación (SUVmax) y tamaño (diámetro máximo tumoral) del tumor pulmonar}

\begin{tabular}{|ccc|}
\hline SUVmax & GGL - & GGL + \\
$<6$ & 9 & 2 \\
$\geq 6$ & 2 & 31 \\
\hline Total & 11 & 33 \\
\hline Fisher & p: 0,000004 & \\
\hline
\end{tabular}

\begin{tabular}{|ccc|}
\hline SUVmax & MTT - & MTT + \\
\hline 6 & 7 & 4 \\
$\geq 6$ & 13 & 20 \\
\hline Total & 20 & 24 \\
\hline Fisher & p: 0,185126 & \\
\hline
\end{tabular}

\begin{tabular}{|ccc|}
\hline SUVmax & GGL - & GGL + \\
$<12$ & 11 & 11 \\
$\geq 12$ & 0 & 22 \\
Total & 11 & 33 \\
Fisher & p: 0,000184 & \\
\hline
\end{tabular}

\begin{tabular}{|ccc|}
\hline SUVmax & MTT - & MTT + \\
$<12$ & 11 & 11 \\
$\geq 12$ & 9 & 13 \\
Total & 20 & 24 \\
Fisher & p: 0,762554 & \\
\hline
\end{tabular}

\begin{tabular}{|ccc|}
\hline Diámetro & GGL - & GGL + \\
$<3 \mathrm{~cm}$ & 9 & 8 \\
$\geq 3 \mathrm{~cm}$ & 2 & 25 \\
Total & 11 & 33 \\
Fisher & $\mathrm{p}: 0,001183$ & \\
\hline
\end{tabular}

\begin{tabular}{|ccc|}
\hline Diámetro & MTT - & MTT + \\
\hline $3 \mathrm{~cm}$ & 12 & 5 \\
$\geq 3 \mathrm{~cm}$ & 8 & 19 \\
\hline Total & 20 & 24 \\
\hline Fisher & $\mathrm{p}: 0,012763$ & \\
\hline
\end{tabular}

\begin{tabular}{|ccc|}
\hline Diámetro & GGL - & GGL + \\
$<5 \mathrm{~cm}$ & 10 & 17 \\
$\geq 5 \mathrm{~cm}$ & 1 & 16 \\
\hline Total & 11 & 33 \\
Fisher & $\mathrm{p}: 0,030854$ & \\
\hline
\end{tabular}

\begin{tabular}{|ccc|}
\hline Diámetro & MTT - & MTT + \\
$<5 \mathrm{~cm}$ & 15 & 12 \\
$\geq 5 \mathrm{~cm}$ & 5 & 12 \\
\hline Total & 20 & 24 \\
\hline Fisher & $\mathrm{p}: 0,124405$ & \\
\hline
\end{tabular}

GGL-: Sin compromiso ganglionar hiliar ni mediastínico; GGL+: Con compromiso hiliar y/o mediastínico; MTT+: con metástasis a distancia; MTT-: sin metástasis a distancia.

predecir la ocurrencia de metástasis con ningún valor de corte, dado que pacientes con tumores de baja captación presentaron metástasis (4 pacientes con adenocarcinoma y SUVmax $<6$ ), y otros con lesiones de alta captación estuvieron libres de éstas ( 9 pacientes con SUVmax $\geq 12$, cinco de ellos con CE).

Los pacientes con tumores de diámetro $\geq 3 \mathrm{~cm}$ presentaron con significativa mayor frecuencia compromiso ganglionar regional y metástasis. El $93 \%$ de los tumores $\geq 3 \mathrm{~cm}(25 / 27)$ y el $94 \%$ de los tumores $\geq 5$ (16/17) mostraron diseminación ganglionar hiliar o mediastínica.

El 50,1\% de los pacientes con cáncer de pulmón falleció (seguimiento promedio: 18 meses, rango: 2-34 meses), con significativa menor sobrevida $(\mathrm{p}<0,05)$ para aquellos con metástasis a distancia, compromiso ganglionar mediastínico, tumor primario con diámetro $\geq 3 \mathrm{~cm}$ y captación tumoral alta $($ SUV $>6)$.

Las curvas de sobrevida según presencia de metástasis y SUVmax se muestran en las Figuras 3 y 4 , respectivamente.

En el análisis de regresión de Cox, sólo la presencia de metástasis pudo predecir mayor mortalidad $(\mathrm{p}<0,001)$.

Excluyendo a los pacientes con metástasis, el estudio de sobreviva de Kaplan-Meier sólo mostró significación para el tipo histológico del cáncer pulmonar, con menor sobrevida para pacientes con CPCP respecto a aquellos con CPCNP (p: 0,0013), como se muestra en la Figura 5. En este grupo de paciente $\mathrm{M} 0$, ningún otro factor fue determinante en el pronóstico. 


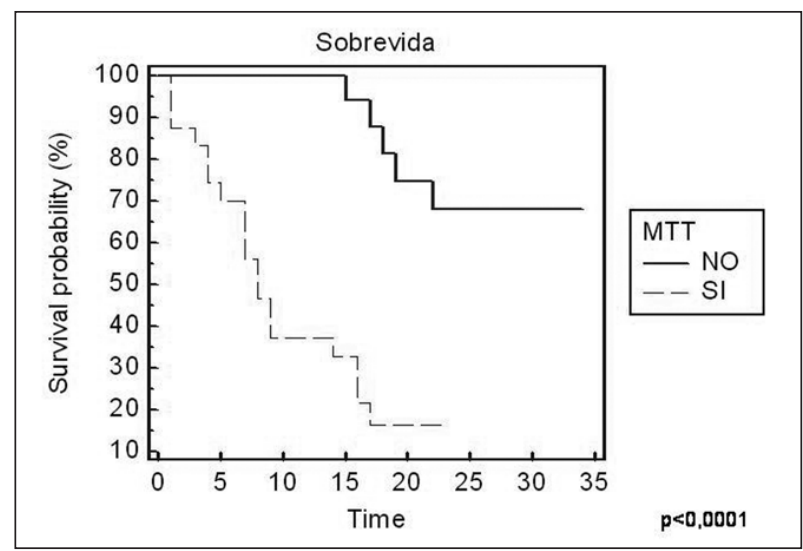

Figura 3. Curva de sobrevida según ocurrencia de metástasis. Los pacientes con diseminación a distancia presentaron significativa menor sobrevida.

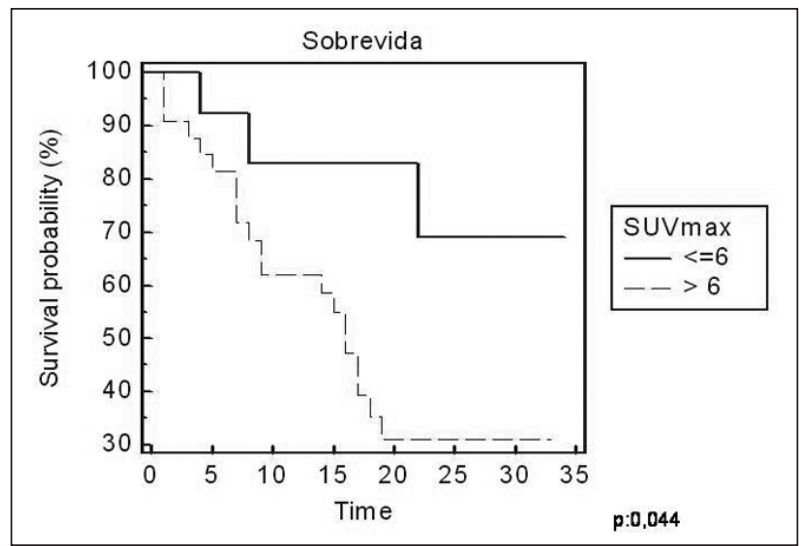

Figura 4. Curva de Kaplan-Meier que muestra menor sobrevida en pacientes con tumores pulmonares con captación alta de F18-FDG (SUVmax >6).

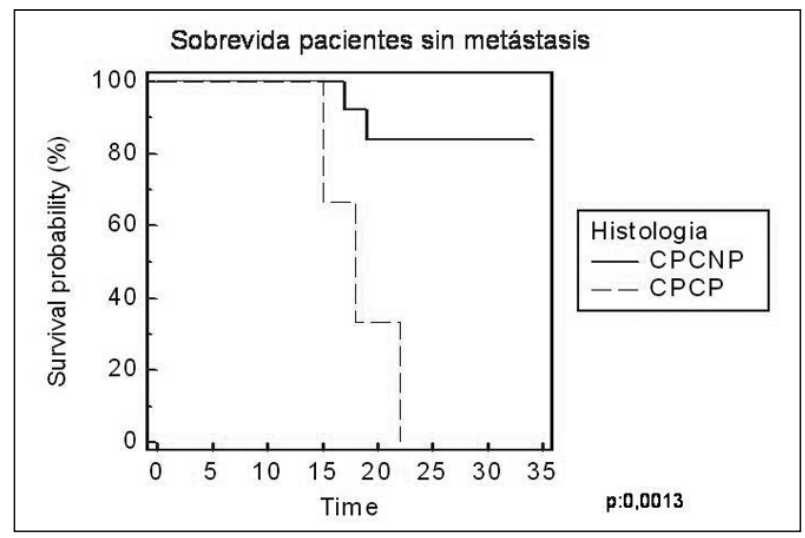

Figura 5. Curva de sobrevida de los pacientes que no presentaron metástasis al momento del PET/CT. En este grupo, los pacientes con CPCP presentaron significativo peor pronóstico que aquellos con CPCNP.

\section{Discusión}

En la actualidad se han identificado más de 150 factores pronósticos en cáncer pulmonar células no pequeñas (CPCNP), siendo el más poderoso y consistente la clasificación $\mathrm{TNM}^{4}$. Recientes publicaciones destacan el valor pronóstico del PET/CT realizado con F18-FDG basado en la asociación entre el grado de captación de glucosa y la "vitalidad" del tumor'. El consumo tumoral de glucosa ha mostrado correlación con el grado de agresividad de la neoplasia, ya que es una expresión de su actividad proliferativa ${ }^{5}$, y por lo tanto, de su crecimiento y capacidad de expandirse, y puede ser cuantificado utilizando índices de captación de los cuales el SUV es el más utilizado ${ }^{6}$.

La posibilidad de cuantificar de manera precisa y reproducible la captación de glucosa en la lesión primaria a través de índices como el SUV ha permitido objetivar su valor pronóstico con la obtención de valores de corte que determinan mejor o peor pronóstico. Por otro lado, la capacidad del PET/ CT de obtener una precisa y confiable etapificación con un solo examen, proporciona importante información adicional sobre el tumor primario y su relación con estructuras vecinas, el compromiso ganglionar hiliar y mediastínico, con un rendimiento mayor al de la TC, y la existencia de metástasis a distancia, sumando estas poderosas variables al análisis pronóstico. Le detección de un segundo tumor primario extrapulmonar por medio de este examen puede ocurrir en $6-19 \%$ de los $\operatorname{casos}^{7-9}$ (4,3\% en nuestra casuística), y sin duda es otro factor que puede modificar el manejo y pronóstico de estos pacientes.

Un metaanálisis publicado recientemente concluye que el SUVmax es un poderosos predictor de sobrevida, con mayor mortalidad en pacientes con tumores pulmonares con alta captación de FDG ${ }^{2}$. Otros estudios añaden que además el $\mathrm{PET} / \mathrm{CT}$ podría identificar a aquellos pacientes que, aun en similar grupo TNM, presentan mayor riesgo de recurrencia y que pudieran ser candidatos de terapia adyuvante ${ }^{10,11}$. Ohtsuka et al observaron la evolución de 98 pacientes 
con adenocarcinoma pulmonar etapa IA y IB operados, donde la recurrencia se observó casi exclusivamente en pacientes con SUVmax mayor a $3,3^{12}$. Tsutani encontró mayor sobrevida libre de enfermedad en pacientes con adenocarcinoma Etapa I con SUV max $\leq 3,7(100 \%)$ respecto a aquellos con SUVmax mayor a 3,7 (57\%) ${ }^{13}$.

El factor pronóstico más poderoso encontrado en nuestra muestra fue la presencia de metástasis, con una clara mayor mortalidad en pacientes que las presentaban. El PET/CT es el método de mayor rendimiento y relación costo/beneficio en la detección de lesiones secundarias, ya que permite explorar todo el cuerpo de una vez con gran sensibilidad. El advenimiento de la técnica híbrida PET/CT ha sido de vital importancia para disminuir los falsos positivos como la "grasa parda" mediastínica, focos inflamatorios pulmonares, fracturas costales y/o vertebrales, y otras situaciones que pueden producir hipercaptación equívoca de F18-FDG, pero que gracias a las imágenes de TC pueden ser fácilmente reconocidas como lesiones benignas ${ }^{6}$.

El tamaño tumoral, el compromiso ganglionar regional y la captación tumoral (SUVmax) también resultaron ser variables determinantes en la sobrevida. Estos tres factores parecen estar ligados estrechamente, dado la correlación encontrada entre tamaño y captación tumoral, y la capacidad del SUVmax y del diámetro tumoral de predecir de manera muy precisa el compromiso ganglionar regional. Todos los pacientes con tumores pulmonares con SUVmax $\geq 12$, y $94 \%$ (31/33) de aquellos con $S U V \max \geq 6$, tuvieron enfermedad ganglionar hiliar y/o mediastínica. Esta asociación entre actividad metabólica del tumor e invasión ganglionar y vascular también ha sido descrita por otros autores ${ }^{13}$.

En aquellos pacientes sin enfermedad metastásica, el tipo histológico tumoral demostró ser la variable pronóstica más poderosa, con menor sobrevida para los CPCP respecto a los CPCNP.

En nuestro estudio se obtuvo una poderosa correlación entre el tamaño tumoral y el índice de captación SUVmax, lo que ha sido descrito en varias otras publicaciones ${ }^{13-16}$, si bien gran parte de éstas han demostrado el valor intrínsico del SUV como variable independiente. Esto también se deduce de nuestro estudio, por cuanto se demuestra una clara diferencia de actividad metabólica entre los tipos histológicos del cáncer pulmonar, sin demostrarse diferencias en sus tamaños. El grado de captación del tumor se relaciona con la sobreexpresión de transportadores de glucosa (GLUT$1)^{17,18}$, y esta sobreexpresión es más frecuente en $\mathrm{CE}(100 \%$ casos) que en adenocarcinoma (58\% casos $)^{19}$. Esto explicaría la marcada diferencia de captación entre adenocarcinomas y CE obtenida en nuestra muestra (SUVmax promedio de 10,3 y 21,0 , respectivamente).

Una de las limitaciones de nuestro trabajo es el relativo bajo número de pacientes incluidos, lo que resulta en una probable subvaloración de factores pronósticos clínicos importantes como la edad, sexo y estado general ${ }^{20}$. Otra limitante es el sesgo en la población estudiada, debido a que la solicitud del PET/CT puede determinar una muestra de individuos con enfermedad particularmente avanzada. Sin embargo, la proporción de pacientes con metástasis y con compromiso ganglionar observada ( $55 \%$ y $25 \%$, respectivamente), es casi idéntica a lo descrito en grandes series con cáncer pulmonar recientemente diagnosticado ${ }^{21,22}$. Por otro lado, la distribución de frecuencia por tipo histológico también es concordante con otros autores, con un predominio de adenocarcinomas, seguido por carcinoma escamoso ${ }^{21}$. Esto puede indicar que la muestra estudiada es representativa de la población general de enfermos de cáncer pulmonar en nuestro medio.

Dado su alto rendimiento y adecuada relación costo-beneficio, el PET/CT es el método no invasivo de elección para etapificar el cáncer pulmonar, y debiera ser realizado en todo paciente con cáncer pulmonar candidato a cirugía ${ }^{22}$.

\section{Conclusiones}

La presencia de metástasis a distancia es el factor pronóstico más poderoso encontrado en cáncer pulmonar, y la mejor manera de detectarlas es por medio del PET/CT.

El grado de captación de F18-FDG por el tumor pulmonar primario mostró gran utilidad pronóstica, con mayor mortalidad en aquellos con SUVmax $>6$. Un diámetro tumoral $\geq 3 \mathrm{~cm}$ y la ocurrencia de compromiso ganglionar regional también determinaron mayor mortalidad.

El índice de captación SUVmax del tumor pulmonar permite predecir su histología y la existencia de compromiso ganglionar hiliar/mediastínico, pero no la diseminación metastásica a distancia. 


\section{Referencias}

1. Dooms C, van Baardwijk A, Verbeken E, van Suylen RJ, Stroobants S, De Ruysscher, et al. Association Between 18F-Fluoro-2-Deoxy-D-Glucose Uptake Values and Tumor Vitality: Prognostic Value of Positron Emission Tomography in Early-Stage Non-small Cell Lung Cancer. J Thorac Oncol 2009; 4: 822-8.

2. Berghmans T, Dusart M, Paesmans M, HosseinFoucher C, Buvat I, Castaigne C, et al. Primary tumor standardized uptake value (SUVmax) measured on fluorodeoxyglucose positron emission tomography (FDG-PET) is of prognostic value for survival in nonsmall cell lung cancer (NSCLC): a systematic review and meta-analysis (MA) by the European Lung Cancer Working Party for the IASLC Lung Cancer Staging Project. J Thorac Oncol 2008; 3: 6-12.

3. Lin EC, Alavi A, Kinahan P. Standarized uptake value. En: Lin EC, Alavi A. PET and PET/CT: A clinical guide. New York, USA: Thieme Medical Publishers Inc; 2005: 28-31.

4. Brundage MD, Davies D and Mackillop WJ. Prognostic factors in non-small cell lung cancer: a decade of progress. Chest 2002; 122: 1037-57.

5. Erasmus JJ, Rohren E and Swisher SG. Prognosis and Reevaluation of Lung Cancer by Positron Emission Tomography. Proc Am Thorac Soc 2009; 6: 171-9.

6. Ladrón de Guevara D, Pefaur R. PET/CT en cáncer pulmonar. Rev Med Chile 2010; 138: 1441-50.

7. Westreenen HL, Westerterp M, Jager PL, van Dullemen HM, Sloof GW, Comans E, et al. Synchronous Primary Neoplasms Detected on 18F-FDG PET in Staging of Patients with Esophageal Cancer. J Nucl Med 2005; 46: 1321-5.

8. Duchateau CSJ, Stokkel MPM. Second Primary Tumors Involving Non-small Cell Lung Cancer. Chest 2005; 127; 1152-8.

9. Lardinois D, Weder W, Roudas M, von Schulthess GK, Tutic M, Moch H, et al. Etiology of Solitary Extrapulmonary Positron Emission Tomography and Computed Tomography Findings in Patients With Lung Cancer. J Clin Oncol 2005; 23: 6846-53.

10. Goodgame B, Pillot GA, Yang Z, Shriki J, Meyers BF, Zoole J, et al. Prognostic value of preoperative positron emission tomography in resected stage I nonsmall cell lung cancer. J Thorac Oncol 2008; 3: 130-4.

11. Hanin FX, Lonneux M, Cornet J, Noirhomme P, Coulon C, Distexhe J, et al. Prognostic value of FDG uptake in early stage nonsmall cell lung cancer. Eur J Cardiothorac Surg 2008; 33: 819-23.

12. Ohtsuka T, Nomori H, Watanabe K, Kaji M, Naruke
T, Suemasu K, et al. Prognostic significance of [(18) F] fluorodeoxyglucose uptake on positron emission tomography in patients with pathologic stage I lung adenocarcinoma. Cancer 2006; 107: 2468-73.

13. Tsutani Y, Miyata Y, Misumi K, Ikeda T, Mimura T, Hihara J, et al. Difference in Prognostic Significance of Maximum Standardized Uptake Value on [18F]-Fluoro2-Deoxyglucose Positron Emission Tomography Between Adenocarcinoma and Squamous Cell Carcinoma of the Lung. Jpn J Clin Oncol 2011; 41: 890-6.

14. Vansteenkiste JF, Stroobants SG, Dupont PJ, De Leyn PR, Verbeken EK, Deneffe GJ, et al. Prognostic importance of the standardized uptake value on (18)F-fluoro-2deoxy-glucose-positron emission tomography scan in non-small-cell lung cancer: an analysis of 125 cases. Leuven Lung Cancer Group. J Clin Oncol 1999; 17: 3201-6.

15. Downey RJ, Akhurst T, Gonen M, Vincent A, Bains MS, Larson S, et al. Preoperative F-18 fluorodeoxyglucosepositron emission tomography maximal standardized uptake value predicts survival after lung cancer resection. J Clin Oncol 2004; 22: 3255-60.

16. Higashi K, Ueda Y, Arisaka Y. 18F-FDG uptake as a biologic prognostic factor for recurrence in patients with surgically resected non-small cell lung cancer. J Nucl Med 2002; 43: 39-45.

17. Mamede $M$, Higashi $T$, Kitaichi M, Ishizu K, Ishimori T, Nakamoto Y, et al. [18F]FDG uptake and PCNA, Glut-1, and Hexokinase-II expressions in cancers and inflammatory lesions of the lung. Neoplasia 2005; 7: 369-79.

18. Nguyen XC, Lee WW, Chung JH, Park SY, Sung SW, Kim YK, et al. FDG uptake, glucose transporter type 1, and Ki-67 expressions in non-small-cell lung cancer: correlations and prognostic values. Eur J Radiol 2007; 62: 214-9.

19. Ito T, Noguchi $Y$, Satoh S, Hayashi H, Inayama Y, Kitamura H. Expression of facilitative glucose transporter isoforms in lung carcinomas: its relation to histologic type, differentiation grade, and tumor stage. Mod Pathol 1998; 11: 437-43.

20. Albain KS, Crowley JJ, LeBlanc M and Livingston RB. Survival determinants in extensive-stage non-small-cell lung cancer: the Southwest Oncology Group experience. J Clin Oncol 1991; 9: 1618-1626.

21. Ettinger DS, Akerley W, Bepler G, Blum MG, Chang A MD, Cheney RT, et al. Non-Small Cell Lung Cancer. Clinical Practice Guidelines in Oncology. J Natl Compr Canc Netw 2010; 8: 740-801.

22. UyBico SJ, Wu CC, Suh RD, Le NH, Brown K, Krishnam MS, et al. Lung Cancer Staging Essentials: The New TNM Staging System and Potential Imaging Pitfalls. RadioGraphics 2010; 30: 1163-81. 